\title{
Proceeding
}

Supplementary Issue: Summer Conferences of Sports Science. Costa Blanca Sports Science Events, 25-26 September 2020. Alicante, Spain.

\section{A systematic review on the muscular activation on the lower limbs with five different variations of the deadlift exercise}

\author{
JORGE FLANDEZ1 ${ }^{1}$, JAVIER GENE-MORALES ${ }^{2,3}$, ALVARO JUESAS ${ }^{3}$, ÁNGEL SAEZ-BERLANGA ${ }^{3}$, IVÁN \\ MIÑANA ${ }^{3}$, JUAN C. COLADO 3 \\ 1 Institute of Education Sciences, Austral University of Chile, Ciudad de Valdivia, Chile \\ ${ }^{2}$ Research Institute on Traffic and Road Safety (INTRAS), University of Valencia, Valencia, Spain \\ ${ }^{3}$ Research Unit in Sport and Health, University of Valencia, Valencia, Spain
}

\begin{abstract}
The deadlift is one of the most widely used resistance exercises by different types of trainees and with different aims including aesthetics, performance and health. There are numerous variations of the deadlift exercise which have different effects on the exercise biomechanics and muscular activation. The main objective of this research was to systematically review the literature looking forward to gathering data on the muscular activation on the lower limbs with different variations of the deadlift exercise (conventional, sumo, Romanian, straight legs and hex-bar). 19 articles met the inclusion criteria and were included in the analysis. Quality of the articles was assessed through the PEDro scale. Main findings were that deadlift is a posteriorchain dominant exercise. Changes in activation between the variations majorly depend on the hip flexion in relation to the knee flexion. For instance, the straight-legs deadlift does not involve knee flexion and mainly activates hip extensors. Other factors such as the distance between the load and the centre of mass, the knee flexion planes, or the total intensity also condition the muscular activation. For example, the hex-bar deadlift allows the subject being in alignment with the load and performing the exercise with relatively vertical back, which provokes an increase on the activation of the knee extensors. In conclusion, this study may help the strength and conditioning professionals and practitioners with the exercise selection depending on the muscular targets and the individual characteristics of the athlete.
\end{abstract}

Keywords: Electromyographic activity; Resistance exercise; Quadriceps; Gluteus; Hamstrings; Calves.

Cite this article as:

Flandez, J., Gene-Morales, J., Juesas, A., Saez-Berlanga, Á., Miñana, I., \& Colado, J.C. (2020). A systematic review on the muscular activation on the lower limbs with five different variations of the deadlift exercise. Journal of Human Sport and Exercise, 15(4proc), S1262-S1276. doi:https://doi.org/10.14198/ihse.2020.15.Proc4.27

Corresponding author. Department of Physical Education and Sports, University of Valencia. C/ Gascó Oliag 3, 46010, Valencia, Spain. https://orcid.org/0000-0002-3255-3940

E-mail: juan.colado@uv.es

Abstract submitted to: Spring Conferences of Sports Science. Costa Blanca Sports Science Events, 19-20 June 2020. Alicante, Spain.

JOURNAL OF HUMAN SPORT \& EXERCISE ISSN 1988-5202

(c) Faculty of Education. University of Alicante

doi:10.14198/jhse.2020.15.Proc4.27

S1262

| 2020 | Proc4 | VOLUME 15

(c) 2020 University of Alicante 


\section{INTRODUCTION}

The benefits of regular physical exercise are well documented (Warburton, 2006). More specifically, adequate resistance training regimens have been shown to promote muscular and systemic adaptations which may be beneficial to manage and prevent certain health conditions such as hypertension, diabetes, osteoporosis (Garber et al., 2011; Stamatakis et al., 2018). Bearing in mind these aforementioned facts, the necessity arises of generating knowledge on the most used resistance training exercises (squat, deadlift, bench press, pull-ups, and military press). In particular, the deadlift is one of the most widely used resistance exercises by different types of trainees and with different aims including aesthetics, performance, and health (Boren et al., 2011; Chen et al., 2018; Lacome et al., 2020; Martín-Fuentes et al., 2020; Neto et al., 2020). This exercise greatly involves the hips (flexion and extension), and thus it has a close relation with different athletic and everyday movements (Bird \& Barrington-Higgs, 2010; González \& Sánchez, 2018). It has been also shown that there is a usual disequilibrium between the quadriceps and hamstrings muscles, which may lead to knee problems (Ruas et al., 2019). In light of these facts, it is worth analysing the muscular activation of the most typically performed lower-limb exercises such as the deadlift.

Muscular activity is often measured with surface electromyography, a method that registers the intensity and duration of electric signals produced in the muscles (Chowdhury et al., 2013). Electrodes are placed on specific superficial points that cover the muscle to analyse. The electromyograph gives raw data in absolute electric signal intensity in millivolts $(\mathrm{mV})$ or microvolts $(\mu \mathrm{V})$. Typical methods to standardize the results are a) as a relative percentage of a maximum voluntary isometric contraction (IMVC); b) as a relative percentage of the maximum historical contraction (MVC); c) as the square root of the average power of the EMG signal for a given period (root mean square; RMS) (Sinclair et al., 2015). Data in the scientific literature are uneven and thus, comparisons between studies are sometimes difficult.

The main objective of this research was to systematically review the expert literature to gather data on the muscular activation of the lower limb during different variants of the deadlift exercise. We aimed to identify the main characteristics of each variant, the predominant muscle groups involved, and to determine the variant with higher activation levels, through the analyses of the included studies.

\section{METHODS}

For this systematic review, the protocols of the PRISMA declaration (Hutton et al., 2015; Urrútia \& Bonfill, 2010) were followed.

\section{Search strategy}

Four databases (Web of Science, PubMed, Scopus, and SportDiscus) and ProQuest (i.e. an electronic tool containing doctoral thesis) were consulted to collect information about muscular activation. Also, the Strength and Conditioning Journal was consulted. No temporal restrictions were used in the search. The following terms were used: ["deadliff" OR "deadlift exercise" OR "conventional deadliff" OR "sumo deadlift" OR "Romanian deadliff" OR "stiff-legs deadliff" OR "RDL" OR "SLDL"] AND ["EMG" OR "electromyography" OR "electromyographic activity" OR "muscle activation" OR "muscle activity"]. The operator "NOT" was used in combination with the terms "balance", "instability", "unstable", "bands", "chains", "injury", "injured", "unload", and "therapeutic" to refine the results and exclude articles that did not follow the inclusion criteria. 


\section{Eligibility criteria}

Studies that examined muscle activation on the lower limb in deadlifts written in Spanish or English were included in the analyses. Inclusion criteria were a) including healthy subjects with no recent history of injury; b) using stable surfaces to perform the deadlifts; c) using a loaded barbell. On the other hand, exclusion criteria were a) using variable resistance (i.e. elastic bands, chains) to load the exercise; b) analysing muscle activity of the upper limb or trunk; c) performing an isometric deadlift.

\section{Article selection and data processing}

\section{Studies}

Potentially relevant studies were selected through the screening of titles and abstracts. The eligibility of each article was defined following the inclusion-exclusion criteria. A standardized form was used to further analyse the studies. Figure 1 shows the study selection process after the reading of the titles and abstracts of the initial results.

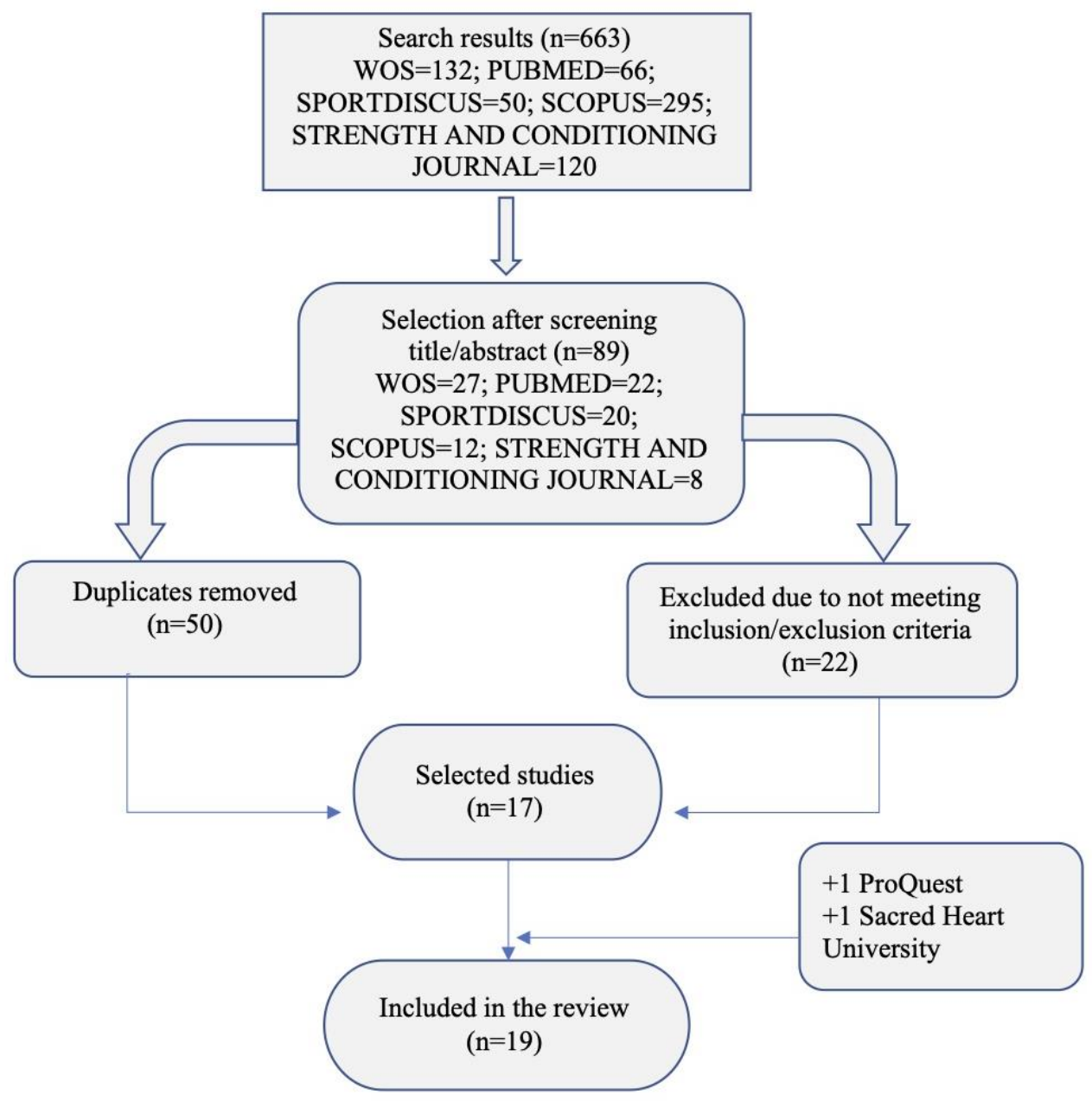

Figure 1. Flow diagram that summarizes the study selection process from the first search to the final selection.

\section{Deadlift variations}

After carefully reading the selected articles five deadlift variations were selected for the analysis by agreement between the authors. The five deadlift variations included were (see Figure 2): 
- Conventional deadlift (12 studies): Stance width should be minor than shoulder-width, feet are close to the centre of the bar, hands are holding the bar per outside the legs (Escamilla et al., 2002).

- Straight legs deadlift ( 6 studies): The same stance as the Romanian deadlift, yet the knees are fully extended. The bar is not in contact with the legs (Lee et al., 2018).

- Romanian deadlift (3 studies): Same stance width as the conventional deadlift. The knee joint angle should not exceed 15 degrees of flexion. The barbell should be in contact with the legs throughout the entire execution (Lee et al., 2018).

- Hex-bar deadlift (2 studies): Same execution as the conventional deadlift. The load distribution presumably changes as the body is inside the hexagon (Andersen et al., 2018).

- Sumo deadlift (1 studies): Wider stance than the conventional deadlift. Stance width is major than shoulder-width (Escamilla et al., 2002).

a)

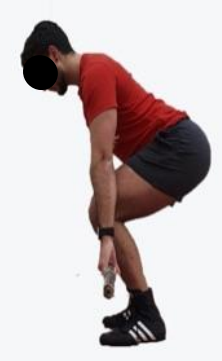

c)

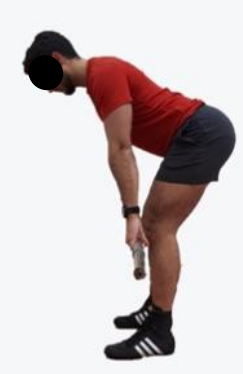

b)

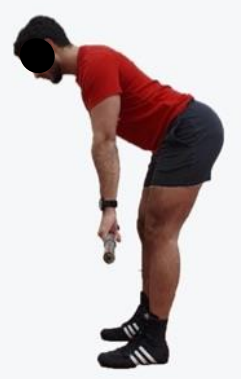

d)

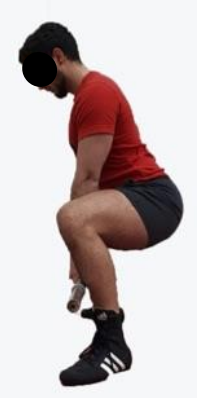

Figure 2. From left to right: a) conventional deadlift, b) straight-legs deadlift, c) Romanian deadlift, d) sumo deadlift. The hex bar deadlift is not pictured as for this study, the position of the body would be the same as in the conventional deadlift.

\section{Muscles analysed}

As mentioned in the objective, this study focuses on the muscles of the lower limb. After a thorough reading of the selected articles, the authors selected the muscles to be included in the analysis. These muscles were a) gluteus maximus, b) gluteus medialis, c) hip adductors, d) vastus lateralis, e) vastus medialis, f) rectus femoris, g) biceps femoris, h) semitendinosus, i) tibialis anterior, j) gastrocnemius and k) soleus. Muscles "a, $b$, and $c$ " act mainly on the hips; muscles " $d, e$, and $f$ ' are part of the quadriceps and are mainly involved in the knee-extension; muscles " $g$ and $h$ " are part of the hamstrings and their contraction mainly affect the knees; the tibialis anterior (muscle " $i$ ") is an ankle dorsiflexor; and finally, the muscles " $j$ and $k$ " are part of the 
calves and their action provoke an ankle extension. Previous expert literature (Netter, 1999) can be consulted for further information on the included muscles and anatomy.

\section{Electromyographic values}

EMG values are unequally reported among the expert literature, not only on the units used (millivolts, microvolts, percentage of isometric maximum voluntary contraction, percentage of maximum voluntary contraction, percentage of root mean square values) but also on the phase measured (concentric and eccentric, mean of the set, mean of a repetition). In this review, the authors standardized the values when possible to facilitate the comprehension and the comparison between studies. For instance, millivolt values were transformed into microvolts. Also, results of concentric and eccentric phases were averaged to obtain a single value.

\section{Quality assessment}

The quality of the included studies was analysed using the PEDro scale (Maher et al., 2003). The scale was modified to fit the design of the included studies (see Table 3). Points 2 and 3 were unified into one point that assessed the randomization of the exercise conditions performed. Point 4 had to be excluded due to not including studies with control and experimental groups. Finally, points 5, 6, and 7 were also excluded due to the impossibility of blinding subjects or researchers. The resultant scale to evaluate the quality of the articles was composed of 6 items.

\section{RESULTS}

19 articles met the inclusion criteria and were reviewed (Figure 1). As mentioned in the "deadlift variations" section, the most widely studied variation of the deadlift exercise was the conventional deadlift (12 studies), followed by the straight-legs deadlift ( 6 studies), the Romanian deadlift ( 3 studies), and hex-bar deadlift ( 2 studies), and finally, only one study analysing the sumo deadlift. The posterior chain is dominant in all the variations, with some differences between each deadlift variation in the involvement of the gluteus, quadriceps, and hamstrings.

Due to the considerable amount of variations and exercise conditions, the results section will be divided into five subsections: one for each deadlift variation. Furthermore, Table 1 includes the main characteristics of the included studies (i.e. sample characteristics, exercise condition, measured muscles, and main results), and Table 2 presents reported EMG values. EMG values are presented in different units (i.e. absolute values, IMVC, MVC, RMS), and thus caution should be applied when comparing values between studies.

\section{Conventional deadlift}

Several studies found the greater activation on the vastus lateralis or even the vastus medialis (Bezerra et al., 2013; Camara et al., 2016; Escamilla et al., 2002; Korak et al., 2018; Nijem et al., 2016; Robbins, 2011; Snyder et al., 2017). Only Fauth et al. (2010) found greater activation on the gluteus maximus compared to the vastus medialis. There is some controversy on which muscle has a greater activity between the gluteus (Escamilla et al., 2002) and biceps femoris (Andersen et al., 2018, 2019; Korak et al., 2018; Lee et al., 2018). Regarding the calf muscles, some authors (Bezerra et al., 2013; Escamilla et al., 2002; Robbins, 2011) found considerable activation on the gastrocnemius and lower activity on the tibialis anterior. Bezerra et al. (2013) reported a considerable high activation also on the tibialis. 
Table 1. Characteristics of the included studies $(N=19)$.

\begin{tabular}{|c|c|c|c|c|c|}
\hline (Author, year) & $\begin{array}{l}\text { Sample (N, sex, age } \\
\text { characteristics) }\end{array}$ & Exercise/s & Load & Measured muscles & Main results \\
\hline $\begin{array}{l}\text { (Andersen et } \\
\text { al., 2019) }\end{array}$ & $\begin{array}{l}15 \text { trained males } \\
\text { Age: } 23.2 \pm 2.2 \text { years } \\
\text { Height: } 182.0 \pm 6.0 \mathrm{~cm} \\
\text { Weight: } 82.8 \pm 11.1 \mathrm{~kg} \text { Experience: } \\
3.9 \pm 1.9 \text { years }\end{array}$ & $\begin{array}{l}\text {-Conventional } \\
\text { deadlift }\end{array}$ & $\begin{array}{l}5 \mathrm{R} \text { at } 85 \% \\
\text { of } 2 \mathrm{RM}\end{array}$ & $\begin{array}{l}\text {-Gluteus maximus } \\
\text {-Vastus lateralis } \\
\text {-Biceps femoris } \\
\text {-Semitendinosus }\end{array}$ & Major activation on the semitendinosus and biceps femoris. \\
\hline $\begin{array}{l}\text { (Delgado et } \\
\text { al., 2019) }\end{array}$ & $\begin{array}{l}8 \text { trained males } \\
\text { Age: } 25.0 \pm 3.3 \text { years } \\
\text { Height: } 177.9 \pm 6.6 \mathrm{~cm} \\
\text { Weight: } 84.0 \pm 6.5 \mathrm{~kg} \\
\text { Experience: } 1 \text { year }\end{array}$ & $\begin{array}{l}\text {-Romanian } \\
\text { deadlift }\end{array}$ & 1RM & $\begin{array}{l}\text {-Gluteus maximus } \\
\text {-Vastus lateralis } \\
\text {-Biceps femoris }\end{array}$ & Major activation on the biceps femoris and gluteus maximus. \\
\hline $\begin{array}{l}\text { (Korak et al., } \\
2018)\end{array}$ & $\begin{array}{l}13 \text { females } \\
\text { Age: } 22.8 \pm 1.0 \text { years } \\
\text { Height: } 166.4 \pm 4.2 \mathrm{~cm} \\
\text { Weight: } 73.4 \pm 14 \mathrm{~kg} \text { Experience: } 1 \\
\text { year }\end{array}$ & $\begin{array}{l}\text {-Conventional } \\
\text { deadlift }\end{array}$ & $\begin{array}{l}3 \mathrm{R} \text { at } \\
75 \% 1 \mathrm{RM}\end{array}$ & $\begin{array}{l}\text {-Gluteus maximus } \\
\text {-Vastus lateralis } \\
\text {-Vastus medialis } \\
\text {-Rectus femoris } \\
\text {-Biceps femoris }\end{array}$ & Major activation on the rectus femoris and vastus lateralis. \\
\hline $\begin{array}{l}\text { (Hegyi et al., } \\
2018 \text { ) }\end{array}$ & $\begin{array}{l}12 \text { males } \\
\text { Age: } 24.3 \pm 3.7 \text { years } \\
\text { Height: } 179.3 \pm 8.8 \mathrm{~cm} \\
\text { Weight: } 74.2 \pm 8.3 \mathrm{~kg}\end{array}$ & $\begin{array}{l}\text {-Straight legs } \\
\text { deadlift }\end{array}$ & $\begin{array}{l}5 \mathrm{R} \text { at } \\
80 \% 1 \mathrm{RM}\end{array}$ & $\begin{array}{l}\text {-Biceps femoris } \\
\text {-Semitendinosus }\end{array}$ & No significant differences between muscles \\
\hline $\begin{array}{l}\text { (McCurdy et } \\
\text { al., 2018) }\end{array}$ & $\begin{array}{l}18 \text { females } \\
\text { Age: } 20.9 \pm 1.1 \text { years } \\
\text { Height: } 165.0 \pm 5.5 \mathrm{~cm} \\
\text { Weight: } 61.84 \pm 6.37 \mathrm{~kg} \\
\text { Experience: } 1 \text { to } 5 \text { years }\end{array}$ & $\begin{array}{l}\text {-Straight legs } \\
\text { deadlift }\end{array}$ & $\begin{array}{l}3 \mathrm{R} \text { at } 8 \mathrm{RM} \\
\text { load }\end{array}$ & $\begin{array}{l}\text {-Gluteus maximus } \\
\text {-Hamstrings }\end{array}$ & Major activation on the gluteus maximus \\
\hline $\begin{array}{l}\text { (Lee et al., } \\
\text { 2018) }\end{array}$ & $\begin{array}{l}21 \text { males } \\
\text { Age: } 22.4 \pm 2.2 \text { years } \\
\text { Height: } 176.0 \pm 7.1 \mathrm{~cm} \\
\text { Weight: } 82.5 \pm 13.0 \mathrm{~kg}\end{array}$ & $\begin{array}{l}\text {-Conventional } \\
\text { deadlift } \\
\text {-Romanian } \\
\text { deadlift }\end{array}$ & $\begin{array}{l}1 \mathrm{R} \text { at } \\
70 \% 1 \mathrm{RM}\end{array}$ & $\begin{array}{l}\text {-Gluteus maximus } \\
\text {-Rectus femoris } \\
\text {-Biceps femoris }\end{array}$ & $\begin{array}{l}\text { Greater general activation in the conventional deadlift. } \\
\text {-Conventional: major activation on the rectus and biceps femoris, } \\
\text { and gluteus maximus, in this order. } \\
\text {-Romanian: major activation on the biceps femoris, gluteus } \\
\text { maximus, and rectus femoris, in this order. }\end{array}$ \\
\hline $\begin{array}{l}\text { (Andersen et } \\
\text { al., 2018) }\end{array}$ & $\begin{array}{l}13 \text { males } \\
\text { Age: } 21.9 \pm 1.6 \text { years } \\
\text { Height: } 180 \pm 5 \mathrm{~cm} \\
\text { Weight: } 81.4 \pm 7.2 \mathrm{~kg}\end{array}$ & $\begin{array}{l}\text {-Conventional } \\
\text { deadlift } \\
\text {-Hex-bar deadlift }\end{array}$ & 1RM & $\begin{array}{l}\text {-Gluteus maximus } \\
\text {-Biceps femoris }\end{array}$ & $\begin{array}{l}\text { Major activation on the biceps femoris using the Olympic barbell and } \\
\text { major activation on the gluteus with the hex-bar. }\end{array}$ \\
\hline
\end{tabular}




\begin{tabular}{|c|c|c|c|c|c|}
\hline $\begin{array}{l}\text { (Iversen et al., } \\
\text { 2017) }\end{array}$ & $\begin{array}{l}12 \text { males and } 12 \text { females } \\
\text { Age: } 25 \pm 3 \text { and } 25 \pm 2 \text { years }\end{array}$ & $\begin{array}{l}\text {-Straight legs } \\
\text { deadlift }\end{array}$ & $\begin{array}{l}3 \mathrm{R} \text { at } \\
\text { 10RM load }\end{array}$ & $\begin{array}{l}\text {-Gluteus maximus } \\
\text {-Vastus lateralis } \\
\text {-Vastus medialis } \\
\text {-Rectus femoris } \\
\text {-Biceps femoris }\end{array}$ & $\begin{array}{l}\text { Major activation on the gluteus maximus, semitendinosus, and } \\
\text { biceps femoris. }\end{array}$ \\
\hline $\begin{array}{l}\text { (Snyder et al., } \\
\text { 2017) }\end{array}$ & $\begin{array}{l}13 \text { males y } 2 \text { females } \\
\text { Age: } 18 \text { to } 24 \text { years }\end{array}$ & $\begin{array}{l}\text {-Conventional } \\
\text { deadlift }\end{array}$ & $\begin{array}{l}\text { 3R at } \\
80 \% 1 \mathrm{RM}\end{array}$ & $\begin{array}{l}\text {-Gluteus maximus } \\
\text {-Vastus lateralis } \\
\text {-Biceps femoris } \\
\end{array}$ & Major activation on the gluteus maximus and vastus lateralis. \\
\hline $\begin{array}{l}\text { (Nijem et al., } \\
2016)\end{array}$ & $\begin{array}{l}13 \text { males } \\
\text { Age: } 24.0 \pm 2.1 \text { years } \\
\text { Height: } 179.3 \pm 4.8 \mathrm{~cm} \\
\text { Weight: } 87 \pm 10.6 \mathrm{~kg}\end{array}$ & $\begin{array}{l}\text {-Conventional } \\
\text { deadlift }\end{array}$ & $\begin{array}{l}3 \mathrm{R} \text { at } \\
85 \% 1 \mathrm{RM}\end{array}$ & $\begin{array}{l}\text {-Gluteus maximus } \\
\text {-Vastus lateralis }\end{array}$ & Major activation on the vastus lateralis than on the gluteus maximus. \\
\hline $\begin{array}{l}\text { (Camara et al., } \\
\text { 2016) }\end{array}$ & $\begin{array}{l}20 \text { males } \\
\text { Age: } 23.3 \pm 2.1 \text { years } \\
\text { Height: } 176.8 \pm 7.6 \mathrm{~cm} \\
\text { Weight: } 89.9 \pm 18.3 \mathrm{~kg}\end{array}$ & $\begin{array}{l}\text {-Conventional } \\
\text { deadlift } \\
\text {-Hex-bar deadlift }\end{array}$ & 1RM & $\begin{array}{l}\text {-Vastus lateralis } \\
\text {-Biceps femoris }\end{array}$ & $\begin{array}{l}\text {-Conventional: low activation on the vastus lateralis. } \\
\text {-Hex-bar: major activation on the vastus lateralis. }\end{array}$ \\
\hline $\begin{array}{l}\text { (Schoenfeld et } \\
\text { al., 2015) }\end{array}$ & $\begin{array}{l}10 \text { males } \\
\text { Age: } 23.5 \pm 3.1 \text { years }\end{array}$ & $\begin{array}{l}\text {-Straight legs } \\
\text { deadlift }\end{array}$ & 8RM & $\begin{array}{l}\text {-Biceps femoris } \\
\text {-Semitendinosus }\end{array}$ & Major activation on the semitendinosus than on the biceps femoris. \\
\hline $\begin{array}{l}\text { (McAllister et } \\
\text { al., 2014) }\end{array}$ & $\begin{array}{l}12 \text { females } \\
\text { Age: } 27 \pm 7 \text { years } \\
\text { Peso: } 78.6 \pm 5.5 \mathrm{~kg}\end{array}$ & $\begin{array}{l}\text {-Romanian } \\
\text { deadlift }\end{array}$ & $\begin{array}{l}1 \mathrm{R} \text { at } \\
85 \% 1 \mathrm{RM}\end{array}$ & $\begin{array}{l}\text {-Gluteus medialis } \\
\text {-Biceps femoris } \\
\text {-Semitendinosus } \\
\text {-Gastrocnemius }\end{array}$ & Major activation on the semitendinosus and biceps femoris. \\
\hline $\begin{array}{l}\text { (Bezerra et al., } \\
\text { 2013) }\end{array}$ & $\begin{array}{l}14 \text { males } \\
\text { Age: } 26.7 \pm 5 \text { year } \\
\text { Height: } 177.7 \pm 8.9 \mathrm{~cm} \\
\text { Weight: } 88.42 \pm 12.39 \mathrm{~kg}\end{array}$ & $\begin{array}{l}\text {-Conventional } \\
\text { deadlift } \\
\text {-Straight legs } \\
\text { deadlift }\end{array}$ & $\begin{array}{l}\text { 1R at } \\
70 \% 1 \mathrm{RM}\end{array}$ & $\begin{array}{l}\text {-Vastus lateralis } \\
\text {-Biceps femoris } \\
\text {-Tibialis anterior } \\
\text {-Gastrocnemius }\end{array}$ & Major activation on the vastus lateralis in the conventional deadlift. \\
\hline $\begin{array}{l}\text { (Robbins, } \\
\text { 2011) }\end{array}$ & $\begin{array}{l}10 \text { males } \\
\text { Age: } 24 \pm 1.18 \text { years } \\
\text { Height: } 177 \pm 5 \mathrm{~cm} \\
\text { Weight: } 82.2 \pm 10.2 \mathrm{~kg}\end{array}$ & $\begin{array}{l}\text {-Conventional } \\
\text { deadlift }\end{array}$ & $\begin{array}{l}3 \mathrm{R} \text { at } \\
85 \% 1 \mathrm{RM}\end{array}$ & $\begin{array}{l}\text {-Gluteus maximus } \\
\text {-Vastus medialis } \\
\text {-Biceps femoris } \\
\text {-Gastrocnemius }\end{array}$ & Major activation on the vastus medialis and the gastrocnemius. \\
\hline $\begin{array}{l}\text { (Fauth et al., } \\
2010)\end{array}$ & $\begin{array}{l}16 \text { females } \\
\text { Age: } 21.19 \pm 2.17 \text { years } \\
\text { Height: } 169.39 \pm 7.54 \mathrm{~cm} \\
\text { Weight: } 66.08 \pm 9.91 \mathrm{~kg}\end{array}$ & $\begin{array}{l}\text {-Conventional } \\
\text { deadlift }\end{array}$ & $\begin{array}{l}2 \mathrm{R} \text { at } 6 \mathrm{RM} \\
\text { load }\end{array}$ & $\begin{array}{l}\text {-Gluteus maximus } \\
\text {-Gluteus medialis } \\
\text {-Vastus lateralis } \\
\text {-Vastus medialis } \\
\text {-Rectus femoris } \\
\text {-Biceps femoris } \\
\text {-Semitendinosus }\end{array}$ & Major activation on the gluteus major and vastus lateralis. \\
\hline
\end{tabular}




\begin{tabular}{|c|c|c|c|c|c|}
\hline $\begin{array}{l}\text { (Ebben et al., } \\
2009)\end{array}$ & $\begin{array}{l}11 \text { males y } 9 \text { females } \\
\text { Age: } 21.5 \pm 1.9 \text { and } 20.0 \pm 1.5 \text { years } \\
\text { Weight: } 78.9 \pm 9.6 \text { and } 66.4 \pm 7.5 \mathrm{~kg}\end{array}$ & $\begin{array}{l}\text {-Conventional } \\
\text { deadlift }\end{array}$ & $\begin{array}{l}2 \mathrm{R} \text { at } 6 \mathrm{RM} \\
\text { load }\end{array}$ & $\begin{array}{l}\text {-Vastus lateralis } \\
\text {-Rectus femoris } \\
\text {-Biceps femoris }\end{array}$ & Major activation on the biceps femoris. \\
\hline $\begin{array}{l}\text { (Escamilla et } \\
\text { al., 2002) }\end{array}$ & $\begin{array}{l}13 \text { males } \\
\text { Age: } 20.1 \pm 1.3 \text { years } \\
\text { Height: } 186.6 \pm 7.5 \mathrm{~cm} \\
\text { Weight: } 102.8 \pm 16.1 \mathrm{~kg}\end{array}$ & $\begin{array}{l}\text {-Conventional } \\
\text { deadlift } \\
\text {-Sumo deadlift }\end{array}$ & 12RM & $\begin{array}{l}\text {-Gluteus maximus } \\
\text {-Hip adductor } \\
\text {-Vastus lateralis } \\
\text {-Vastus medialis } \\
\text {-Rectus femoris } \\
\text {-Hamstrings } \\
\text {-Tibialis anterior } \\
\text {-Gastrocnemius }\end{array}$ & $\begin{array}{l}\text { Significantly major activation on the vastus lateralis and medialis, } \\
\text { and the tibialis anterior in the sumo deadlift compared to the } \\
\text { conventional deadlift. Major activation on the gastrocnemius in the } \\
\text { conventional deadlift. }\end{array}$ \\
\hline $\begin{array}{l}\text { (Wright et al., } \\
\text { 1999) }\end{array}$ & $\begin{array}{l}6 \text { football players and } 5 \\
\text { bodybuilders }\end{array}$ & $\begin{array}{l}\text {-Straight legs } \\
\text { deadlift }\end{array}$ & $\begin{array}{l}3 \mathrm{R} \text { at } \\
75 \% 1 \mathrm{RM}\end{array}$ & $\begin{array}{l}\text {-Biceps femoris } \\
\text {-Semitendinosus }\end{array}$ & No significant differences were found between muscles. \\
\hline
\end{tabular}

Note: Age, height, weight, and experience values are presented as Mean \pm Standard Deviation. EMG: Electromyography; R: Repetitions; RM: Repetition Maximum.

Table 2. Electromyographic activity reported in each study.

\begin{tabular}{|c|c|c|c|c|c|c|c|c|c|c|c|c|}
\hline \multirow{2}{*}{$\begin{array}{l}\text { (Author, } \\
\text { year) }\end{array}$} & \multirow{2}{*}{$\begin{array}{l}\text { EMG } \\
\text { value }\end{array}$} & \multirow[t]{2}{*}{ Deadlift } & \multicolumn{10}{|c|}{ Measured muscles } \\
\hline & & & GM & GMed & $A D$ & VL & VM & $\mathrm{RF}$ & $\mathrm{BF}$ & ST & TA & GN \\
\hline $\begin{array}{l}\text { (Andersen } \\
\text { et al., 2019) }\end{array}$ & $\begin{array}{l}\text { Raw } \\
(\mu \mathrm{V})\end{array}$ & $\begin{array}{l}\text { Conventional } \\
\text { deadlift }\end{array}$ & 236 & - & - & 239 & - & - & 312 & 367 & - & - \\
\hline $\begin{array}{l}\text { (Delgado et } \\
\text { al., 2019) }\end{array}$ & $\begin{array}{l}\text { Raw } \\
(\mu \mathrm{V})\end{array}$ & $\begin{array}{l}\text { Romanian } \\
\text { deadlift }\end{array}$ & $\sim 160$ & - & - & $\sim 100$ & - & - & $\sim 200$ & - & - & - \\
\hline $\begin{array}{l}\text { (Korak et } \\
\text { al., 2018) }\end{array}$ & $\begin{array}{l}\% \\
\text { MVC }\end{array}$ & $\begin{array}{l}\text { Conventional } \\
\text { deadlift }\end{array}$ & $\sim 72$ & - & - & $\sim 104$ & $\sim 92$ & $\sim 105$ & $\sim 82$ & - & - & - \\
\hline $\begin{array}{l}\text { (Hegyi et } \\
\text { al., 2018) }\end{array}$ & $\begin{array}{l}\% \\
\text { IMVC }\end{array}$ & $\begin{array}{l}\text { Straight legs } \\
\text { deadlift }\end{array}$ & - & - & - & - & - & - & $\sim 36$ & $\sim 37$ & - & - \\
\hline $\begin{array}{l}\text { (McCurdy } \\
\text { et al., 2018) }\end{array}$ & $\begin{array}{l}\% \\
\text { IMVC }\end{array}$ & $\begin{array}{l}\text { Straight legs } \\
\text { deadlift }\end{array}$ & $40.5 \pm 19.2$ & - & - & - & - & - & Hamstring & $9 \pm 14.0$ & & - \\
\hline \multirow[t]{2}{*}{$\begin{array}{l}\text { (Lee et al., } \\
\text { 2018) }\end{array}$} & $\begin{array}{l}\% \\
\text { RMS }\end{array}$ & $\begin{array}{l}\text { Conventional } \\
\text { deadlift }\end{array}$ & - & - & & - & - & $58.7 \pm 13.7$ & $57.5 \pm 6.3$ & - & - & - \\
\hline & & $\begin{array}{l}\text { Romanian } \\
\text { deadlift }\end{array}$ & $46.9 \pm 7.4$ & - & - & - & - & $25.3 \pm 14.2$ & $56.7 \pm 18.6$ & - & - & - \\
\hline \multirow[t]{2}{*}{$\begin{array}{l}\text { (Andersen } \\
\text { et al., 2018) }\end{array}$} & $\begin{array}{l}\% \% \\
\text { IMVC }\end{array}$ & $\begin{array}{l}\text { Conventional } \\
\text { deadlift }\end{array}$ & $\sim 95$ & - & - & - & - & - & $\sim 108$ & - & - & - \\
\hline & & $\begin{array}{l}\text { Hex-bar } \\
\text { deadlift }\end{array}$ & $\begin{array}{l}>88 \\
\end{array}$ & - & - & - & - & - & $\begin{array}{l}83 \\
\end{array}$ & - & - & - \\
\hline $\begin{array}{l}\text { (Iversen et } \\
\text { al., 2017) }\end{array}$ & $\begin{array}{l}\% \\
\text { MVC }\end{array}$ & $\begin{array}{l}\text { Straight legs } \\
\text { deadlift }\end{array}$ & $\sim 31$ & - & - & $\sim 16$ & $\sim 11$ & $\sim 7$ & $\sim 29$ & $\sim 30$ & - & - \\
\hline
\end{tabular}




\begin{tabular}{|c|c|c|c|c|c|c|c|c|c|c|c|c|}
\hline $\begin{array}{l}\text { (Snyder et } \\
\text { al., 2017) }\end{array}$ & $\begin{array}{l}\% \\
\text { RMS }\end{array}$ & $\begin{array}{l}\text { Conventional } \\
\text { deadlift }\end{array}$ & $\sim 47$ & - & - & $\sim 48$ & - & - & $\sim 28$ & - & - & - \\
\hline $\begin{array}{l}\text { (Nijem et } \\
\text { al., 2016) }\end{array}$ & $\begin{array}{l}\% \\
\text { MVC }\end{array}$ & $\begin{array}{l}\text { Conventional } \\
\text { deadlift }\end{array}$ & $82 \pm 6$ & - & - & $115 \pm 30$ & - & - & - & - & - & - \\
\hline \multirow[t]{2}{*}{$\begin{array}{l}\text { (Camara et } \\
\text { al., 2016) }\end{array}$} & \multirow[t]{2}{*}{$\begin{array}{l}\text { MVC } \\
\text { MVC }\end{array}$} & $\begin{array}{l}\text { Conventional } \\
\text { deadlift }\end{array}$ & - & - & - & $76.4 \pm 7.3$ & - & - & $59.1 \pm 15.0$ & - & - & - \\
\hline & & $\begin{array}{l}\text { Hex-bar } \\
\text { deadlift }\end{array}$ & - & - & - & $103.9 \pm 26.5$ & - & - & $51.9 \pm 15$ & - & - & - \\
\hline $\begin{array}{l}\text { (Schoenfeld } \\
\text { et al., 2015) } \\
\end{array}$ & $\begin{array}{l}\% \\
\text { IMVC }\end{array}$ & $\begin{array}{l}\text { Straight legs } \\
\text { deadlift }\end{array}$ & - & - & - & - & - & - & 58 & 85 & - & - \\
\hline $\begin{array}{l}\text { (McAllister } \\
\text { et al., 2014) }\end{array}$ & $\begin{array}{l}\text { Raw } \\
(\mu \mathrm{V})\end{array}$ & $\begin{array}{l}\text { Romanian } \\
\text { deadlift }\end{array}$ & - & $\sim 190$ & - & - & - & - & $\sim 330$ & $\sim 800$ & - & $\sim 190$ \\
\hline \multirow[t]{2}{*}{$\begin{array}{l}\text { (Bezerra et } \\
\text { al., 2013) }\end{array}$} & \multirow[t]{2}{*}{$\begin{array}{l}\% \\
\text { RMS }\end{array}$} & $\begin{array}{l}\text { Conventional } \\
\text { deadlift }\end{array}$ & - & - & - & $128.3 \pm 33.9$ & - & - & $100.1 \pm 24.7$ & - & $104.0 \pm 18.8$ & - \\
\hline & & $\begin{array}{l}\text { Straight legs } \\
\text { deadlift }\end{array}$ & - & - & - & $100.1 \pm 14.6$ & - & - & $98.6 \pm 28.5$ & - & $109.2 \pm 15.3$ & - \\
\hline $\begin{array}{l}\text { (Robbins, } \\
\text { 2011) }\end{array}$ & $\begin{array}{l}\% \\
\text { MVC }\end{array}$ & $\begin{array}{l}\text { Conventional } \\
\text { deadlift }\end{array}$ & $\sim 45$ & - & - & - & $\sim 65$ & - & $\sim 40$ & - & - & $\sim 61$ \\
\hline $\begin{array}{l}\text { (Fauth et } \\
\text { al., 2010) }\end{array}$ & $\begin{array}{l}\% \\
\text { IMVC }\end{array}$ & $\begin{array}{l}\text { Conventional } \\
\text { deadlift }\end{array}$ & $128 \pm 62$ & $41 \pm 22$ & - & $62 \pm 30$ & $105 \pm 51$ & $54 \pm 49$ & $92 \pm 62$ & $67 \pm 36$ & - & - \\
\hline $\begin{array}{l}\text { (Ebben et } \\
\text { al., 2009) }\end{array}$ & $\begin{array}{l}\% \\
\text { IMVC }\end{array}$ & $\begin{array}{l}\text { Conventional } \\
\text { deadlift }\end{array}$ & - & - & - & $\sim 59$ & - & $\sim 39$ & $\sim 58$ & - & - & - \\
\hline \multirow[t]{2}{*}{$\begin{array}{l}\text { (Escamilla } \\
\text { et al., 2002) }\end{array}$} & \multirow[t]{2}{*}{$\begin{array}{l}\% \\
\text { IMVC }\end{array}$} & $\begin{array}{l}\text { Conventional } \\
\text { deadlift }\end{array}$ & $35 \pm 27$ & - & $24 \pm 22$ & $40 \pm 22$ & $36 \pm 25$ & $19 \pm 16$ & $28 \pm 19$ & $27 \pm 23$ & $13 \pm 8$ & $31 \pm 21$ \\
\hline & & Sumo deadlift & $37 \pm 28$ & - & $23 \pm 16$ & $48 \pm 24$ & $44 \pm 27$ & $18 \pm 13$ & $29 \pm 19$ & $31 \pm 23$ & $18 \pm 9$ & $27 \pm 20$ \\
\hline $\begin{array}{l}\text { (Wright et } \\
\text { al. 1999) }\end{array}$ & $\begin{array}{l}\% \\
\text { MVC }\end{array}$ & $\begin{array}{l}\text { Straight legs } \\
\text { deadlift }\end{array}$ & - & - & - & - & - & - & $67.2 \pm 16.0$ & $68.0 \pm 16.3$ & - & - \\
\hline
\end{tabular}

Values are expressed as Mean \pm Standard Deviation or as a percentage. EMG: Electromyography; GM: Gluteus Maximus; GMed: Gluteus Medialis; HA: Hip Adductors; VL: Vastus Lateralis; VM: Vastus Medialis; RF: Rectus Femoris; BF: Biceps Femoris; ST: Semitendinosus; TA: Tibialis Anterior; GN: Gastrocnemius; SL: Soleus; \%IMCV: percentage of an isometric maximum voluntary contraction; \%MVC: percentage of the historic maximum voluntary contraction; \%RMS: percentage of peak root mean square (RMS); $\mu \mathrm{V}$ : microvolts. 
Table 3. Quality assessment of the included studies.

\begin{tabular}{|c|c|c|c|c|c|c|c|}
\hline Author (year) & 1 & 2 & 3 & 4 & 5 & 6 & Total \\
\hline Andersen et al. (2019) & + & + & + & + & + & + & 6 \\
\hline Delgado et al. (2019) & + & + & + & + & + & + & 6 \\
\hline Korak et al. (2018) & + & + & + & + & + & + & 6 \\
\hline Hegyi et al. (2018) & + & + & + & + & + & + & 6 \\
\hline McCurdy et al. (2018) & + & + & + & + & + & + & 6 \\
\hline Lee et al. (2018) & + & + & + & + & + & + & 6 \\
\hline Andersen et al. (2018) & + & + & + & + & + & + & 6 \\
\hline Iversen et al. (2017) & + & - & + & + & + & + & 5 \\
\hline Snyder et al. (2017) & + & + & + & + & + & + & 6 \\
\hline Nijem et al. (2016) & + & + & + & + & + & + & 6 \\
\hline Camara et al. (2016) & + & + & + & + & + & + & 6 \\
\hline Schoenfeld et al. (2015) & + & + & + & + & + & + & 6 \\
\hline McAllister et al. (2014) & + & + & + & + & + & + & 6 \\
\hline Bezerra et al. (2013) & + & - & + & + & + & + & 5 \\
\hline Robbins (2011) & + & + & + & + & + & + & 6 \\
\hline Fauth et al. (2010) & + & + & + & + & + & + & 6 \\
\hline Ebben et al. (2009) & + & + & + & + & + & + & 6 \\
\hline Escamilla et al. (2002) & + & + & + & + & + & + & 6 \\
\hline Wright et al. (1999) & + & + & + & + & + & + & 6 \\
\hline
\end{tabular}

\section{Straight legs deadlift}

In this variation, there is a more notable activity of the posterior chain in comparison to the quadriceps. The controversy lies on the difference in the activation between the semitendinosus and the biceps femoris, with some authors (Hegyi et al., 2018; Iversen et al., 2017; McCurdy et al., 2018; Schoenfeld et al., 2015) reporting higher values on the semitendinosus, and one study (Wright et al., 1999) higher, but not significant, values on the hamstrings. The studies comparing the activation on the gluteus maximus and the hamstrings in this deadlift variation (Iversen et al., 2017; McCurdy et al., 2018) found a higher activity on the gluteus. Bezerra et al. (2013) reported a considerable high activation on the tibialis also in this variation of the deadlift exercise.

\section{Romanian deadlift}

The biceps femoris was the main involved muscle followed by the gluteus and the quadriceps (Delgado et al., 2019; Lee et al., 2018). However, one study which compared the activation on the semitendinosus, biceps femoris, and gluteus medialis, found significantly higher activation on the semitendinosus (McAllister et al., 2014). These authors reported a considerable high activity on the gastrocnemius.

\section{Hex-bar deadlift}

A slightly higher activation on the gluteus maximus compared to the biceps femoris was found in one study (Andersen et al., 2018). The other study analysing the hex-bar deadlift reported a higher activation on the vastus lateralis in comparison to the biceps femoris (Camara et al., 2016).

\section{Sumo deadlift}

The only study analysing this deadlift variation (Escamilla et al., 2002) concluded that the main muscles involved are the vastus lateralis and medialis, followed by the gluteus maximus. Also, these authors reported a considerable activation on the gastrocnemius, which was greater than the activation on the hamstrings. 


\section{DISCUSSION}

The main objectives of this bibliographic research were to identify the main muscle group involved in the execution of the deadlift and the variation with the higher activation levels. The primary most remarkable finding is that although the deadlift is a posterior-chain dominant exercise (Andersen et al., 2018; Delgado et al., 2019; Fauth et al., 2010; Hegyi et al., 2018; Iversen et al., 2017; Lee et al., 2018; McAllister et al., 2014; McCurdy et al., 2018; Schoenfeld et al., 2015; Wright et al., 1999), considerable levels of muscle activity were found on the vastus lateralis and medialis (Bezerra et al., 2013; Camara et al., 2016; Escamilla et al., 2002; Korak et al., 2018; Nijem et al., 2016; Robbins, 2011; Snyder et al., 2017). These changes in activation majorly depend on the ratio knee-hip flexion as have been observed between the different variations of the deadlift (i.e. conventional, straight legs, Romanian, hex bar, and sumo).

In this regard, when the hips remain higher than the knees during the execution of the deadlift (e.g. in the straight-leg and Romanian deadlifts), fewer degrees of knee flexion is required (see Figure 2). Due to this, the trunk becomes more horizontal than in movements involving greater knee flexion, and consequently, the effort falls mainly on hip-extensor muscles like the gluteus and also the hamstrings (Delgado et al., 2019; Lee et al., 2018). Conversely in the variations involving higher degrees of knee flexion (e.g. in the sumo and conventional deadlifts) major activation is enhanced on the knee-extensor muscles (Bezerra et al., 2013; Camara et al., 2016; Escamilla et al., 2002; Korak et al., 2018; Nijem et al., 2016; Robbins, 2011; Snyder et al., 2017). Reinforcing this hypothesis, the comparison between the conventional and sumo deadlift unveils differences in the activation patterns, with the sumo deadlift provoking greater activation on the knee extensors than the conventional deadlift (Escamilla et al., 2002). In the sumo deadlift a higher knee flexion is achieved compared to the conventional deadlift (see Figure 2).

It is also worth highlighting the different activation patterns observed between the conventional deadlift performed with an Olympic barbell or a hex-bar. Even though they are executed with the same joint positioning, the displacement of the load in relation to the mass centre of the body has been shown to affect muscle activity. With the hex-bar, the load is situated at both sides of the subject, and this causes a major activation on the knee extensors compared to the use of an Olympic barbell (Camara et al., 2016). With the Olympic barbell, the load is located in front of the subject. The effort seems that is shared between the hips and knees when using the Olympic barbell (Andersen et al., 2018; Camara et al., 2016).

Bearing in mind that under the same volume, higher loads entail higher muscle activation levels (Aspe \& Swinton, 2014; McBride et al., 2002), it is necessary to evaluate the movements under a biomechanical perspective to understand the variation with higher activation levels. As previously reported (see "deadlift variations" section) in the sumo and conventional deadlift the bar is in contact with the body, and the centre of mass of the body moves altogether with the bar (i.e. hips going down as the bar is going down). Contrariwise, in the straight-leg deadlift and also in the Romanian deadlift the hips remain higher when the bar goes down. In these variations, the barbell is ousted from the body mass centre, and thus the lever angle increases. This fact limits the opportunity of using higher loads in these two variations and converts them into posterior-chain analytic exercises (Delgado et al., 2019; Iversen et al., 2017). Something similar happens between the sumo and conventional deadlifts and also between the conventional deadlift performed with the Olympic bar or with the hex-bar. As previously reported, in the sumo deadlift and also in the hex-bar deadlift the trunk remains vertical compared to the conventional deadlift. Thus, the lever arm is more favourable and higher loads can be used (Escamilla et al., 2002). Due to these abovementioned facts, the sumo deadlift is the variation in which higher loads can be used, and thus, higher activation levels may be achieved. This variation would be followed, in terms of general activation levels, by the conventional deadlift. Finally, the 
Romanian and straight-leg deadlifts would provoke lower levels of general activation due to the less favourable biomechanical conditions and the limited opportunity of using high loads.

Activity on the calf muscles was found to be important in all the analysed variations. The gastrocnemius was the main involved calf muscle, followed by the tibialis anterior (Bezerra et al., 2013; Escamilla et al., 2002; McAllister et al., 2014; Robbins, 2011). None of the included studies analysed the activity on the soleus. It is worth mentioning that a study aimed at comparing muscle activation between squats and deadlift found higher activation levels on the calves with the deadlift (Robbins, 2011). Escamilla et al. (2002) reported higher gastrocnemius activation in the conventional deadlift and higher tibialis anterior activation in the sumo deadlift.

\section{Limitations}

The included studies have some limitations that should be listed. In this regard, none of the studies indicate what type of isometric contraction (e.g. pushing or holding; Schaefer \& Bittmann, 2017) performed the subjects to obtain the isometric maximum voluntary contraction to standardize the EMG results. In this line, it is worth mentioning that standardization values are uneven, and this may entail a problem when trying to compare results and extract conclusions. Also, one study found significant differences between the proximal and distal fibres of the biceps femoris and semitendinosus (Schoenfeld et al., 2015). These differences between fibre bundles of the same muscle may condition the EMG results. Finally, and even all the procedures of the present review were carefully carried out it is not free of limitations. The standardization made in the values by the authors may limit the analysis of each phase of the execution (i.e. eccentric and concentric). Future studies should review the literature comparing the activation in each phase. Also, the disparities between the included studies may carry to limited comparisons and extraction of conclusions. Finally, our inclusion criteria did not include variable resistance or unstable surfaces. These factors may provide the strength and conditioning professionals and athletes to further understanding of deadlift exercises.

\section{CONCLUSION}

This study highlights the importance of studying the neuromuscular acute effects of the deadlift to deeply understand the exercise and its variations and individualize resistance exercise programs. In brief, we observed that the deadlift is a posterior-chain dominant exercise. Different variations entailed different activation patterns and activation levels. The changes in activation patterns were found to be dependent on the hip and knee flexion ratio. The evidence presented in this study may help the strength and conditioning professionals and practitioners with the exercise selection depending on the muscular targets and the individual characteristics of the athlete.

\section{REFERENCES}

Andersen, V., Fimland, M. S., Mo, D.-A., Iversen, V. M., Larsen, T. M., Solheim, F., \& Saeterbakken, A. $H$. (2019). Electromyographic comparison of the barbell deadlift using constant versus variable resistance in healthy, trained men. PloS One, 14(1), e0211021. https://doi.org/10.1371/journal.pone.0211021

Andersen, V., Fimland, M. S., Mo, D.-A., Iversen, V. M., Vederhus, T., Rockland Hellebø, L. R., Nordaune, K. I., \& Saeterbakken, A. H. (2018). Electromyographic comparison of barbell deadlift, hex bar deadlift, and hip thrust exercises: A cross-over study. Journal of Strength and Conditioning Research, 32(3), 587-593. https://doi.org/10.1519/JSC.0000000000001826 
Aspe, R. R., \& Swinton, P. A. (2014). Electromyographic and kinetic comparison of the back squat and overhead squat. Journal of Strength and Conditioning Research, 28(10). https://doi.org/10.1519/JSC.0000000000000462

Bezerra, E. S., Simao, R., Fleck, S. J., Paz, G., Maia, M., Costa, P. B., Amadio, A. C., Miranda, H., \& Serrao, J. C. (2013). Electromyographic activity of lower body muscles during the deadlift and stilllegged deadlift. Journal of Exercise Physiology Online, 16(3), 30-40.

Bird, S., \& Barrington-Higgs, B. (2010). Exploring the deadlift. Strength and Conditioning Journal, 32(2), 46-51. https://doi.org/10.1519/SSC.0b013e3181d59582

Boren, K., Conrey, C., Coguic, J. L., Paprocki, L., Voight, M., \& Robinson, T. K. (2011). Electromyographic analysis of gluteus medius and gluteus maximus during rehabilitation exercises. International Journal of Sports Physical Therapy, 6(3), 206.

Camara, K. D., Coburn, J. W., Dunnick, D. D., Brown, L. E., Galpin, A. J., \& Costa, P. B. (2016). An examination of muscle activation and power characteristics while performing the deadlift exercise with straight and hexagonal barbells. Journal of Strength and Conditioning Research, 30(5), 11831188. https://doi.org/10.1519/JSC.0000000000001352

Chen, C. H., Chen, Y. S., Wang, Y. T., Tseng, W. C., \& Ye, X. (2018). Effects of preconditioning hamstring resistance exercises on repeated sprinting-induced muscle damage in female soccer players. Biology of Sport, 35(3), 269. https://doi.org/10.5114/biolsport.2018.77827

Delgado, J., Drinkwater, E. J., Banyard, H. G., Haff, G. G., \& Nosaka, K. (2019). Comparison between back squat, romanian deadlift, and barbell hip thrust for leg and hip muscle activities during hip extension. Journal of Strength and Conditioning Research, 33(10), 2595-2601. https://doi.org/10.1519/JSC.0000000000003290

Ebben, W. P., Feldmann, C. R., Dayne, A., Mitsche, D., Alexander, P., \& Knetzger, K. J. (2009). Muscle activation during lower body resistance training. International Journal of Sports Medicine, 30(1), 1-8. https://doi.org/10.1055/s-2008-1038785

Escamilla, R. F., Francisco, A. C., Kayes, A. V., Speer, K. P., \& Moorman, C. T. (2002). An electromyographic analysis of sumo and conventional style deadlifts. Medicine and Science in Sports and Exercise, 34(4), 682-688. https://doi.org/10.1249/00005768-200204000-00019

Fauth, M. L., Garceau, L. R., Lutsch, B., Gray, A., Szalkowski, C., Wurm, B., \& Ebben, W. P. (2010). Hamstrings, quadriceps, and gluteal muscle activation during resistance training exercises. Proceedings of the 28th Conference of the International Society of Biomechanics in Sports, 195-198.

Garber, C. E., Blissmer, B., Deschenes, M. R., Franklin, B. A., Lamonte, M. J., Lee, I.-M., Nieman, D. C., \& Swain, D. P. (2011). Quantity and quality of exercise for developing and maintaining cardiorespiratory, musculoskeletal, and neuromotor fitness in apparently healthy adults: Guidance for prescribing exercise. Medicine \& Science in Sports \& Exercise, 43(7), 1334-1359. https://doi.org/10.1249/MSS.0b013e318213fefb

González, J. R., \& Sánchez, J. S. (2018). Métodos de entrenamiento de la fuerza para la mejora de las acciones en el fútbol [Strength Training Methods for Improving Actions in Football]. Apunts. Educación física y deportes, 2(132), 72-93.

Hegyi, A., Péter, A., Finni, T., \& Cronin, N. J. (2018). Region-dependent hamstrings activity in Nordic hamstring exercise and stiff-leg deadlift defined with high-density electromyography. Scandinavian Journal of Medicine \& Science in Sports, 28(3). https://doi.org/10.1111/sms.13016

Iversen, V. M., Mork, P. J., Vasseljen, O., Bergquist, R., \& Fimland, M. S. (2017). Multiple-joint exercises using elastic resistance bands vs. conventional resistance-training equipment: A cross-over study. $\begin{array}{llll}\text { European Journal of } & \text { Sport } & \text { Science, } & 17(8),\end{array}$ https://doi.org/10.1080/17461391.2017.1337229 
Korak, J. A., Paquette, M. R., Fuller, D. K., Caputo, J. L., \& Coons, J. M. (2018). Muscle activation patterns of lower-body musculature among 3 traditional lower-body exercises in trained women. Journal of Strength and Conditioning Research, 32(10), 2770-2775. https://doi.org/10.1519/JSC.0000000000002513

Lacome, M., Avrillon, S., Cholley, Y., Simpson, B. M., Guilhem, G., \& Buchheit, M. (2020). Hamstring Eccentric Strengthening Program: Does training volume matter? International Journal of Sports Physiology and Performance, 15(1), 81-90. https://doi.org/10.1123/ijspp.2018-0947

Lee, S., Schultz, J., Timgren, J., Staelgraeve, K., Miller, M., \& Liu, Y. (2018). An electromyographic and kinetic comparison of conventional and Romanian deadlifts. Journal of Exercise Science and Fitness, 16(3), 87-93. https://doi.org/10.1016/j.jesf.2018.08.001

Martín-Fuentes, I., Oliva-Lozano, J. M., \& Muyor, J. M. (2020). Electromyographic activity in deadlift exercise and its variants. A systematic review. PLoS ONE, 15(2), e0229507. https://doi.org/10.1371/journal.pone.0229507

McAllister, M. J., Hammond, K. G., Schilling, B. K., Ferreria, L. C., Reed, J. P., \& Weiss, L. W. (2014). Muscle activation during various hamstring exercises. Journal of Strength and Conditioning Research, 28(6), 1573-1580. https://doi.org/10.1519/JSC.0000000000000302

McBride, J. M., Triplett-McBride, T., Davie, A., \& Newton, R. U. (2002). The effect of heavy- vs. Lightload jump squats on the development of strength, power, and speed. Journal of Strength and Conditioning Research, 16(1), 75-82. https://doi.org/10.1519/00124278-200202000-00011

McCurdy, K. W., Walker, J. L., \& Yuen, D. (2018). Gluteus maximus and hamstring activation during selected weight-bearing resistance exercises. Journal of Strength and Conditioning Research, 32(3), 594-601. https://doi.org/10.1519/JSC.0000000000001893

Neto, W. K., Soares, E. G., Vieira, T. L., Aguiar, R., Chola, T. A., Sampaio, V. de L., \& Gama, E. F. (2020). Gluteus maximus activation during common strength and hypertrophy exercises: A systematic review. Journal of Sports Science \& Medicine, 19(1), 195-203.

Netter, F. H. (1999). Human Anatomy. Elsevier - Health Sciences Division.

Nijem, R. M., Coburn, J. W., Brown, L. E., Lynn, S. K., \& Ciccone, A. B. (2016). Electromyographic and force plate analysis of the deadlift performed with and without chains. Journal of Strength and Conditioning Research, 30(5), 1177-1182. https://doi.org/10.1519/JSC.0000000000001351

Robbins, D. (2011). A comparison of muscular activation during the back squat and deadlift to the countermovement jump [Master Thesis, Sacred Heart University].

Ruas, C. V., Pinto, R. S., Haff, G. G., Lima, C. D., \& Brown, L. E. (2019). Effects of different combinations of concentric and eccentric resistance training programs on traditional and alternative hamstrings-toquadriceps ratios. Sports, 7(10), 221. https://doi.org/10.3390/sports7100221

Schaefer, L. V., \& Bittmann, F. N. (2017). Are there two forms of isometric muscle action? Results of the experimental study support a distinction between a holding and a pushing isometric muscle function. BMC Sports Science, Medicine and Rehabilitation, 9(1), 11. https://doi.org/10.1186/s13102-017$\underline{0075-z}$

Schoenfeld, B. J., Contreras, B., Tiryaki-Sonmez, G., Wilson, J. M., Kolber, M. J., \& Peterson, M. D. (2015). Regional differences in muscle activation during hamstrings exercise. Journal of Strength and Conditioning Research, 29(1), 159-164. https://doi.org/10.1519/JSC.0000000000000598

Snyder, B. J., Cauthen, C. P., \& Senger, S. R. (2017). Comparison of muscle involvement and posture between the conventional deadlift and a 'walk-In' style deadlift machine. Journal of Strength and Conditioning Research, 31(10), 2859-2865. https://doi.org/10.1519/JSC.0000000000001723

Stamatakis, E., Lee, I.-M., Bennie, J., Freeston, J., Hamer, M., O'Donovan, G., Ding, D., Bauman, A., \& Mavros, Y. (2018). Does strength-promoting exercise confer unique health benefits? A pooled analysis of data on 11 population cohorts with all-cause, cancer, and cardiovascular mortality 


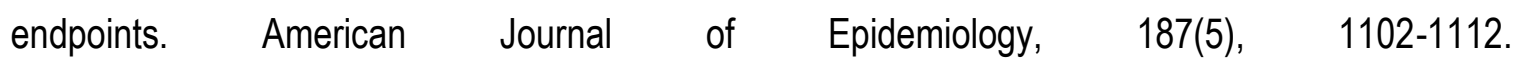
https://doi.org/10.1093/aje/kwx345

Warburton, D. E. R. (2006). Health benefits of physical activity: The evidence. Canadian Medical Association Journal, 174(6), 801-809. https://doi.org/10.1503/cmaj.051351

Wright, G. A., Delong, T. H., \& Gehlsen, G. (1999). Electromyographic activity of the hamstrings during performance of the leg curl, stiff-leg deadlift, and back squat movements. Journal of Strength and Conditioning Research, 13(2), 168-174. https://doi.org/10.1519/00124278-199905000-00012 